\title{
Comparing the oscillation phase in optical pump-probe spectra to ultrafast $x$-ray diffraction in the metal-dielectric $\mathrm{SrRuO}_{3} / \mathrm{SrTiO}_{3}$ superlattice
}

\author{
André Bojahr, ${ }^{1}$ Daniel Schick, ${ }^{1}$ Lena Maerten, ${ }^{1}$ Marc Herzog, ${ }^{1}$ Ionela Vrejoiu, ${ }^{2}$ Clemens von Korff Schmising, ${ }^{3}$ Chris Milne, ${ }^{4}$ \\ Steven L. Johnson, ${ }^{5}$ and Matias Bargheer ${ }^{1,6, *}$ \\ ${ }^{1}$ Institute of Physics and Astronomy, University of Potsdam, Karl-Liebknecht-Strasse 24-25, 14476 Potsdam, Germany \\ ${ }^{2}$ Max-Planck-Institut für Mikrostrukturphysik, Weinberg 2, 06120 Halle, Germany \\ ${ }^{3}$ Institut für Optik und Atomare Physik, TU Berlin, Strasse des 17. Juni 135, 10623 Berlin \\ ${ }^{4}$ Swiss Light Source, Paul Scherrer Institut, 5232 Villigen PSI, Switzerland and Laboratoire de Spectroscopie Ultrarapide, \\ Ecole Polytechnique Federale de Lausanne, 1015 Lausanne, Switzerland \\ ${ }^{5}$ Institute for Quantum Electronics, ETH Zurich, Wolfgang-Pauli-Strasse 16, 8093 Zurich, Switzerland \\ ${ }^{6}$ Helmholtz Zentrum Berlin, Albert-Einstein-Strasse 15, 12489 Berlin, Germany \\ (Received 6 April 2012; revised manuscript received 16 May 2012; published 19 June 2012)
}

\begin{abstract}
We measured the ultrafast optical response of metal-dielectric superlattices by broadband all-optical pumpprobe spectroscopy. The observed phase of the superlattice mode depends on the probe wavelength, making assignments of the excitation mechanism difficult. Ultrafast $\mathrm{x}$-ray diffraction data reveal the true oscillation phase of the lattice which changes as a function of the excitation fluence. This result is confirmed by the fluence dependence of optical transients. We set up a linear chain model of the lattice dynamics and successfully simulated the broadband optical reflection by unit-cell resolved calculation of the strain-dependent dielectric functions of the constituting materials.
\end{abstract}

DOI: 10.1103/PhysRevB.85.224302

PACS number(s): 63.20.Ry, 42.65.Es

\section{INTRODUCTION}

Optical femtosecond spectroscopy is an established tool to infer ultrafast dynamics in molecules and solids. Time constants such as the exponential decay or the oscillation period can often be directly ascribed to microscopic processes such as relaxation or vibration. A detailed modeling of the ultrafast optical response requires the quantum-chemical modeling of molecular potentials in the case of molecular systems or calculation of the dielectric function of solids. With the broader accessibility of various experimental setups for ultrafast $\mathrm{x}$-ray diffraction (UXRD), the findings from alloptical experiments can be cross-checked by directly looking at the lattice motion, including a real time measurement of the absolute atomic amplitudes. In the case of bulk semiconductors UXRD allowed conclusions to be drawn on modifications of the strain fronts induced by the fast diffusion of hot carriers. ${ }^{1-3}$ For bulk bismuth UXRD in combination with ab initio simulations revealed how the lattice potential changes with the time-dependent carrier density. ${ }^{4}$ UXRD and ultrafast electron-diffraction studies show that, for increasing excitation fluence, electronic pressure gains importance versus phonon pressure in metallic systems. ${ }^{5,6}$ All these processes influence the oscillation phase of the excited phonons. In reverse, measuring the oscillation phase elucidates the excitation mechanisms.

The influence of Raman excitation has been discussed in bulk systems, superlattices, and multilayers. ${ }^{7}$ Under strictly nonresonant conditions this excitation causes a sine-like phase of the lattice motion. In contrast the so-called displacive excitation of coherent phonons (DECP) ${ }^{8}$ results in a cosine-like lattice motion. ${ }^{9}$ DECP is exclusively observed in opaque materials and can be described by the imaginary part of the Raman tensor. ${ }^{10}$ Additional excitation mechanisms which show a displaced equilibrium of the lattice oscillation are surface charge screening, the photo Dember effect, or heating of the lattice by rapid electron-phonon scattering. ${ }^{11}$ In many cases the oscillation phase was measured by alloptical techniques and ascribed to the phase of the lattice motion. Raman scattering with real and imaginary tensor contributions is held responsible for the excitation of coherent phonons in metallic systems $(\mathrm{Cd}, \mathrm{Zn}, \mathrm{Zr})$ as well. ${ }^{12,13} \mathrm{In}$ the most intensively investigated material, the semimetal $\mathrm{Bi}$, the microscopic interpretation of the excitation is developed in detail. Theory predicts the time-dependent change of the interatomic potential during the relaxation of photoexcited carriers. ${ }^{4,14}$ A similarly detailed interpretation of the excitation in terms of quasiparticle generation is exemplified for $\mathrm{Si}^{15}$ UXRD would yield direct experimental information on the lattice motion in this case. However, there is only a single UXRD experiment that measures lattice dynamics (polaritons) induced by nonresonant Raman excitation. ${ }^{16}$

Several all-optical studies measured a fluence dependent phase of oscillations, which was ascribed to the simultaneous action of DECP and Raman mechanisms. ${ }^{17}$ Superlattices exhibit phonon modes which are very well suited for fundamental tests, as their periods can be tuned via the layer thickness. Especially for UXRD experiments they yield high signal-tonoise ratio of the experimental signal. The nanosized layers support zone folded acoustic phonons ${ }^{18}$ with few-picosecond oscillation periods, well suited for distinguishing tiny phase differences. Optical excitation of the opaque constituent of a superlattice yields a standing strain wave where the opaque material is periodically expanded while the transparent material is compressed. For GaAs/AlGaAs superlattices a UXRD study under high fluence conditions revealed a dominant DECP mechanism ${ }^{19}$ whereas all-optical measurements under lowfluence conditions suggest the Raman mechanism. ${ }^{7}$ Although it is well established to use such ultrafast structural techniques, the problem of determining the arrival time of the $\mathrm{x}$-ray or 
electron pulses with high precision relative to the pump pulse persists. A direct and detailed comparison of ultrafast optical response over a broad spectral range with the actual lattice motion determined by ultrafast diffraction techniques under identical excitation conditions is lacking.

In this contribution we revisit the metal-dielectric superlattice (SL) composed of $\mathrm{SrRuO}_{3}$ (SRO) and $\mathrm{SrTiO}_{3}$ (STO), for which it was shown by UXRD measurements that there are at least two contributing mechanisms for photoexcited lattice dynamics: thermal expansion by electron-phonon and phonon-phonon interaction and ultrafast magnetostriction. ${ }^{21}$ We present a fluence-dependent UXRD study which shows a relative phase shift of about 130 fs of the lattice motion, indicating that for high fluence the photon energy is coupled more rapidly to the expansion. At high fluences the measured phase of the lattice motion is consistent with an instantaneous DECP mechanism within the experimental error. In broadband optical reflectivity measurements we find that the oscillation phase of the all-optical signal strongly depends on the probe wavelength; however, we can confirm the relative shift of the oscillation phase with fluence. To determine the absolute phase of the lattice oscillation we perform a combined optical pump-probe and UXRD experiment without moving any components.

In order to better understand the broadband optical response, the lattice dynamics are calculated in a massesand-springs model and calibrated in phase and amplitude using the UXRD data. The calculated spatiotemporal strain pattern is used to simulate the optical response by using the strain-dependent dielectric functions of SRO and STO, where the dependence $\partial N_{\mathrm{SRO}} / \partial \eta$ of the complex refractive index $N_{\text {SRO }}$ on the strain $\eta$ is the only freely adjustable parameter. The agreement of these $\mathrm{x}$-ray calibrated simulations with the optical response is very good. These data directly show that all-optical pump-probe data can exhibit nearly arbitrary oscillation phases, even if the lattice dynamics are fixed.

\section{SETUP AND EXPERIMENTAL RESULTS}

The sample consists of 10 double layers of STO/SRO $(13 \mathrm{~nm} / 7.5 \mathrm{~nm})$ deposited by pulsed laser deposition on an STO substrate. ${ }^{22}$ In all experiments presented in this manuscript we use pump pulses with a wavelength of $\lambda_{\text {pump }}=$ $800 \mathrm{~nm}$. Figure 1(a) presents fluence-dependent UXRD data recorded at the MicroXAS-FEMTO beamline of the Swiss Light Source (SLS). ${ }^{23}$ The amplitude of the oscillation has been analyzed ${ }^{24}$ and discussed previously. ${ }^{20}$ The according strain amplitude is reproduced in Fig. 1(b). Panel 1(c) shows the delay $t_{0}^{o s c}$ of the oscillation phase extracted from fitting the data in panel 1(a) to an analytical function [Eq. (1)] describing the sample response, which will be further discussed in Sec. IV. In short, the symmetric superlattice-phonon mode of the metallic/insulating superlattice is exited by expanding the metal layers. For the highest fluence the oscillation starts approximately 130 fs earlier. Figure 2(a) shows the transient optical reflectivity of the sample at $\lambda_{\text {probe }}=670 \mathrm{~nm}$. The rapid rise of the reflectivity originates from quasi-instantaneous heating of the metal electrons. The rising edge determines the arrival time of the optical pump pulse. The slanted arrows indicate the delay of the oscillation for lower pump fluence. For
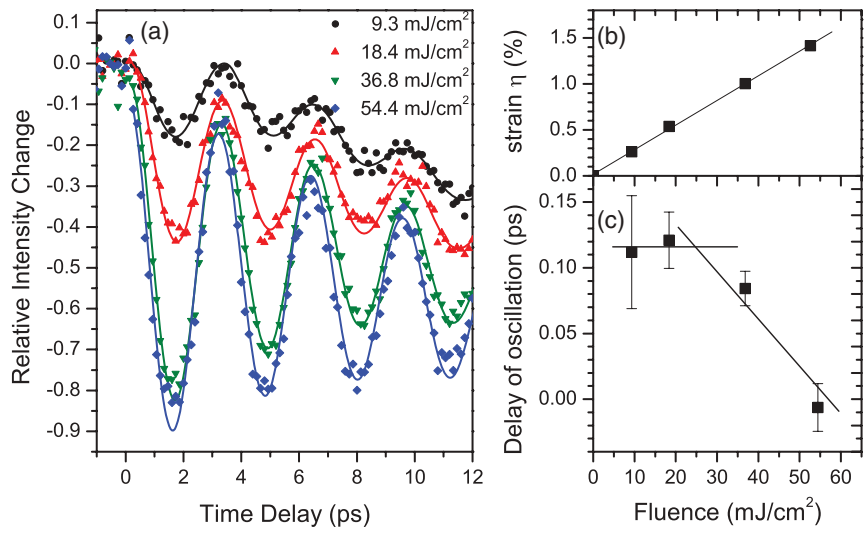

FIG. 1. (Color online) (a) UXRD measurements of the $\left(\begin{array}{llll}0 & 0 & 116\end{array}\right)$ reflection of the SRO/STO SL recorded at the SLS $\lambda_{\text {pump }}=800 \mathrm{~nm}$ for different fluences. (b) Strain amplitude derived from the data in panel (a) by comparison to a dynamical x-ray diffraction simulation (Ref. 20). (c) Oscillation phase extracted from the measured data using the fit function of eq. 1 . The error bars correspond to a $68 \%$ confidence interval from fitting the relative phase. The absolute phase is obtained by comparison to the experiments at the laser-based plasma source discussed in Fig. 4.

an accurate evaluation we subtract the incoherent background (dotted line) and fit oscillations to the data. The resulting linear fluence dependence of the amplitude is shown in Fig. 2(b). The phase delay is plotted in Fig. 2(c) and compared to the UXRD result. The excellent agreement suggests that indeed for low fluence the oscillation is delayed by about 130 fs due to a finite electron-phonon coupling time as the expansion is dominated by phonon-phonon interaction. In contrast, for high fluence the
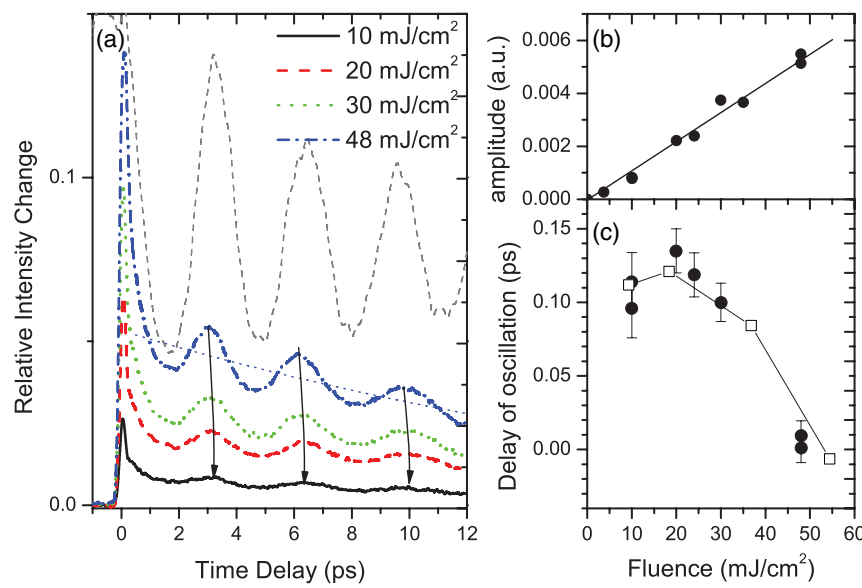

FIG. 2. (Color online) (a) Measured optical reflectivity $\lambda_{\text {probe }}=$ $670 \mathrm{~nm}$ for different fluences. The dotted line indicates the incoherent background contribution which is subtracted from each transient to fit the oscillations and to plot Fig. 3(a). The thin dashed line shows the UXRD measurement for comparison. (b) Oscillation amplitude as a function of the fluence. (c) Comparison of the relative oscillation phase of the optical signal (solid circles) to the phase determined by UXRD [open squares reproduced from Fig. 1(c)]. The error bars of the relative phase are determined from the maximum deviation of phases in two measurement series. The absolute phase is shifted to agree with the UXRD data. 

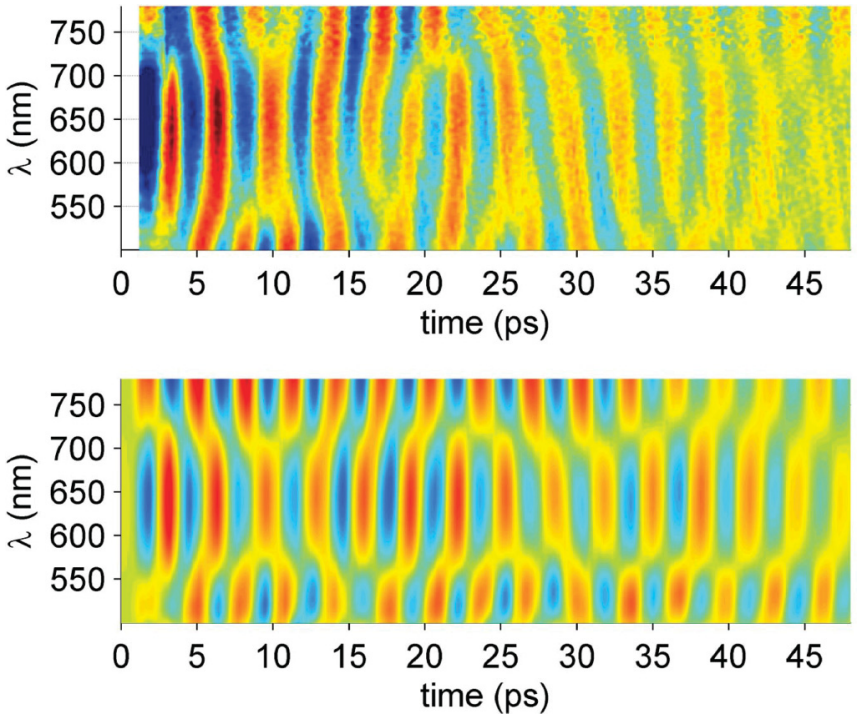

FIG. 3. (Color) (a) Experimentally measured change of the optical reflectivity from $\lambda_{\text {probe }}=500$ to $780 \mathrm{~nm}$ at normal incidence after exciting the sample at $\lambda_{\text {pump }}=800 \mathrm{~nm}$ as a function of time delay. The electronic response and a slowly varying signal contribution have been subtracted as exemplified in Fig. 2(a). (b) Results from the simulation described in the text. The slowly varying background has been subtracted as well. The simulations show that the spectral position of the phase change at 560 and $720 \mathrm{~nm}$ is given by an interference of light reflected from the surface and the interface to the substrate.

time for coupling the energy into the expansion mode is so fast that we cannot distinguish it from an instantaneous response.

Our broadband optical pump-probe setup is similar to those reported in the literature, ${ }^{25,26}$ where a white-light continuum serves as the probe pulse. Hence, we not only measure the data at $670 \mathrm{~nm}$ (Fig. 2) but over the full visible range. The analysis teaches us to interpret all-optical data with great care. After subtraction of the slowly varying background for each wavelength as exemplified by the dotted line in Fig. 2(a), the broadband data exhibit complex dependence of the phase on the probe wavelength [Fig. 3(a)].

A general problem in UXRD experiments is the precise determination of the time zero. For all-optical pump-probe data we cross-checked that the rising edge of the signal corresponds to $t=0$ by sum-frequency generation of pump and probe in a beta-Barium-Borate (BBO) crystal. To calibrate the time origin of the UXRD data, we repeated the UXRD experiment at the laser-based femtosecond diffractometer at the University of Potsdam. ${ }^{27}$ We removed the x-ray optic which is used for the standard operation of the diffractometer. ${ }^{27,28}$ The Bragg condition selects a small angular range of the generated $\mathrm{x}$-ray pulses which are diffracted from the sample (hatched beam in the schematic in Fig. 4). We introduce slits along this x-ray beam to ensure that, after removing the copper tape for $\mathrm{x}$-ray generation, only laser photons propagating along this $\mathrm{x}$-ray probe path impinge on the sample, now as optical probe pulses.

To switch between optical and x-ray probes, only the copper band and a $10 \mu \mathrm{m}$ thick plastic film are removed from the beam path. The optical probe pulse and the $\mathrm{x}$-ray probe-pulse have

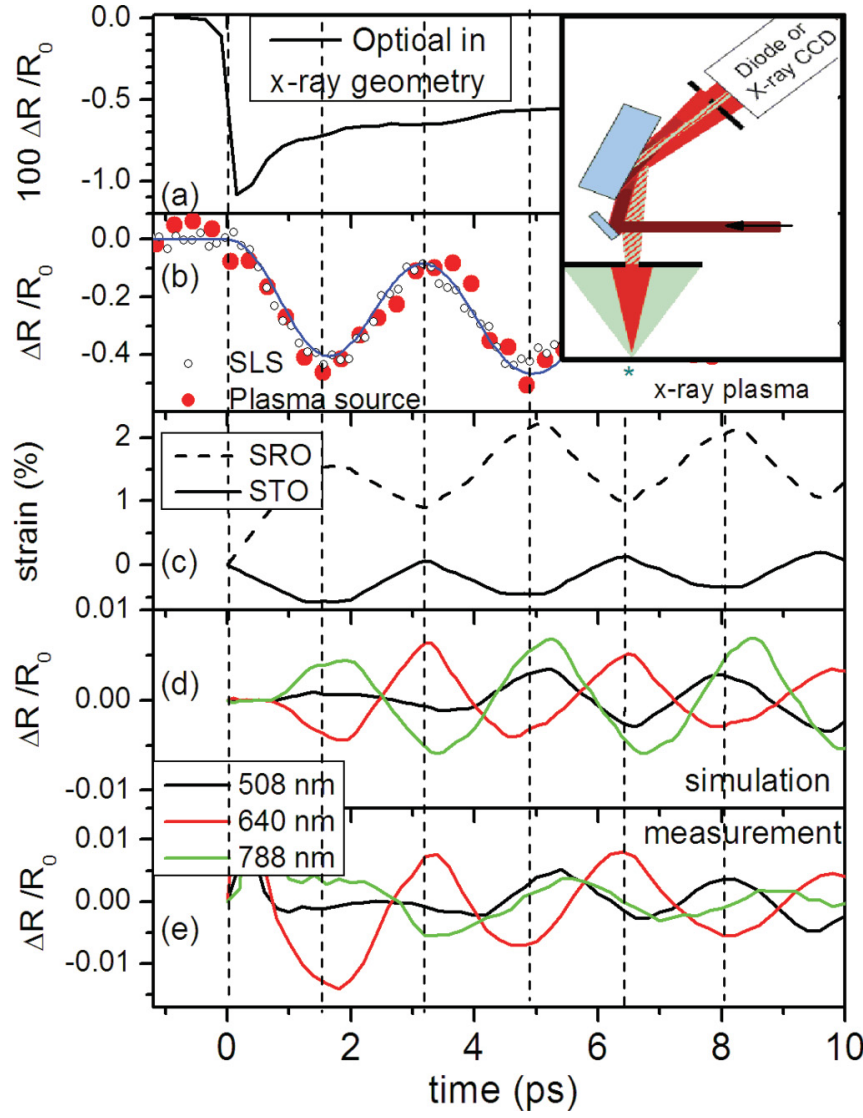

FIG. 4. (Color) (a) All-optical response measured in the same configuration for $\lambda_{\text {pump }}=800 \mathrm{~nm}$ and $\lambda_{\text {probe }}=800 \mathrm{~nm}$. The schematic shows the combined optical/x-ray pump-probe geometry. Optical (red) and x-ray (green) probe pulses collinearly propagate through a slit towards the sample. Both emerge from the laser focus on the copper tape which generates the $\mathrm{x}$-ray pulses and which is removed for optical probing. (b) UXRD data from the laser-based plasma source (red dots) with removed $\mathrm{x}$-ray optics to precisely determine the zero time delay. The signal is shifted by $250 \mathrm{fs}$ to earlier times with respect to the data as measured, according to the analysis described in the text. For comparison we show the UXRD data measured at the SLS [open circles, reproduced from Fig. 1(a)]. The blue line is the simulated UXRD signal based on the calculated lattice dynamics. (c) Simulated average strain in STO (solid) and SRO (dashed) pattern which is consistent with the observed $\mathrm{x}$-ray diffraction signal. (d) Simulated optical reflectivity for three selected probe wavelengths. (e) Corresponding color-coded horizontal cuts through Fig. 3(a).

the same geometric path. The optical path is different due to the decreased group velocities $c^{*}$ in air for x rays $\left(c_{\mathrm{xray}}^{*}-c\right) / c<$ $10^{-6}$ and $800 \mathrm{~nm}$ light pulses $\left(c_{\mathrm{opt}}^{*}-c\right) / c=3 \times 10^{-4}$. The $300 \mathrm{~mm}$ path in air behind the laser focus makes the $\mathrm{x}$ rays arrive 300 fs earlier than the laser pulse traveling the same path in the all-optical experiment. In addition the laser passes through $5 \mathrm{~cm}$ of vacuum before the focus in the x-ray experiment, which is replaced by an air path in the optical experiment, adding another 50 fs to the time delay. A contribution in the opposite direction is the additional time delay of the $\mathrm{x}$-ray pulses due to the propagation of electrons in the metal target before the $\mathrm{x}$-ray production. This leads to a temporally extended x-ray 
pulse with a duration in the 100-200 fs range ${ }^{29}$ as compared to the $40 \mathrm{fs}$ laser pulses. In essence this delays the $\mathrm{x}$-ray pulse by about $100 \mathrm{fs}$, since the leading edge of the x-ray pulse must coincide with the optical excitation pulse as the first generated $x$ rays travel at the speed of light, and the trailing edge is delayed by the $\mathrm{x}$-ray pulse duration which is given by the hot electrons moving through the copper target. ${ }^{30}$

In short, the time axis of the UXRD experiment must be shifted by $250 \mathrm{fs}$ with respect to the all-optical experiment. Time zero is determined by the steepest slope due to the electronic heating in SRO detected in the all-optical experiment [Fig. 4(a)], which is consistent with the time overlap determined in a thin BBO crystal for second-harmonic generation. Now the UXRD signal is measured in exactly the same configuration and plotted in Fig. 4(b) already shifted as discussed above. For comparison the data from Fig. 1(a) measured at the SLS are shown as well. Panel 4(c) compares this to simulations discussed below, which also predict the optical response for three selected wavelengths [panel 4(d)], which are in excellent agreement with the measured data at these wavelengths [panel 4(e)]. The pump fluence in both the UXRD and the optical experiment was approximately $20 \mathrm{~mJ} / \mathrm{cm}^{2}$. In total, Fig. 4 summarizes how time zero is compared in all-optical and UXRD experiments and how both data sets are linked to the simulation of lattice dynamics discussed in the next section.

Earlier UXRD measurements on the very same STO/SRO SL reported an additional delay of approximately $500 \mathrm{fs}$ for weak excitation. ${ }^{21,31,32}$ Very careful analysis of all experiments shows that this discrepancy can be partly ascribed to a modified oscillation phase for the higher pump fluence and partly to the group-velocity delay of the optical pulses and x-ray pulses in air which was neglected at that time.

\section{SIMULATIONS}

As a theoretical support of our interpretations, we have set up a linear chain model of the superlattice in which instantaneous stress is generated by optically induced heating of the lattice. The elastic constants are known and the spatiotemporal strain pattern has been previously calculated. ${ }^{33}$ On the timescale $t<10$ ps heat diffusion can be neglected and it is not relevant for the stress generation. ${ }^{34}$ In the optical signal it gives rise to a slowly varying background that is subtracted before considering the oscillations. The average strain in the SRO and STO layers resulting from this calculation is given in Fig. 4(c). The simulated strain map with unit-cell resolution is the common starting point to predict both the UXRD signal [panel 4(b)] and the all-optical signal [panel 4(d)]. The simulated solid line in panel 4(b) is obtained from using the calculated spatio-temporal strain pattern ${ }^{33}$ in a dynamical $\mathrm{X}$-ray diffraction simulation. ${ }^{20}$

To calculate the optical response we specify the complex index of refraction $N(z, t)=n+i \kappa$ for each unit cell along growth direction of the superlattice for each timestep and calculate the optical reflectivity in a standard optical matrix formalism. For STO both the wavelength-dependent index of refraction $n_{\mathrm{STO}}(\lambda)$ and its derivative $d n_{\mathrm{STO}} / d \eta=0.5$ with respect to strain $\eta$ are taken from the literature. ${ }^{35-37}$ The complex refractive index $N_{\mathrm{SRO}}(\lambda)=n_{\mathrm{SRO}}(\lambda)+i \kappa_{\mathrm{SRO}}(\lambda)$ of SRO is derived from the literature. ${ }^{38}$ The derivatives for SRO are unknown and hence we use $d n_{\mathrm{SRO}} / d \eta$ and $d \kappa_{\mathrm{SRO}} / d \eta$ as fitting parameters to match the observed data simultaneously for all probe wavelengths. We already obtain a reasonable agreement [compare Figs. 3(a) and 3(b) and Figs. 4(d) and 4(e)] if we assume $d n_{\mathrm{SRO}} / d \eta=d n_{\mathrm{STO}} / d \eta=0.5$ and $d \kappa_{\mathrm{SRO}} / d \eta=1.5$ independent of wavelength. Additional variations of the optical constants with the wavelength could yield even more accurate agreement. However, already at the current level of simulation the agreement of the optical broadband data with simulations presented in Fig. 3(b) gives us confidence in our interpretation. From an experimental point of view the validation via UXRD is a preferable cross-check.

\section{DISCUSSION}

For $t>0$ the UXRD signals can be well fitted by the following function:

$S(t)=-A \sin \left(\pi \frac{t-t_{0}^{\mathrm{osc}}}{T_{\mathrm{osc}}}\right)^{2} \exp \left(-\frac{t-t_{0}^{\mathrm{osc}}}{T_{\mathrm{dec}}}\right)-m\left(t-t_{0}^{\mathrm{lin}}\right)$.

The shape of this fitting function is uniquely determined by the physics contained in the simulation. The oscillatory part originates from the excitation of a single zone-folded LA phonon mode which modulates the $\mathrm{x}$-ray diffraction structure factor of the superlattice reflection, and the decay is essentially due to the propagation of the excitation into the substrate. ${ }^{20}$ The linear slope on the signal is explained by the shift of the Bragg reflection due to the average heat expansion of the superlattice.

Such a simple fitting function does not exist for the all-optical counterpart. Figure 3 illustrates that the optical signals suffer from a beating due to the interference of probe pulses reflected from the interfaces, from the phonons in the SL, and from the propagating sound wave. Despite this complicated situation, the straightforward simulation yields excellent agreement.

Now we turn to the discussion of the phase shift observed in both experiments. From the carefully determined time zero of the UXRD signal with an accuracy of about $\pm 100 \mathrm{fs}$, we can directly conclude that the assumption of an instantaneous stress generation in our simulation is very good, since the phase of the signal at the highest fluence corresponds to a perfect cosine, i.e., to a displacive excitation without considerable additional delay due to electron-phonon coupling. The phase of the lattice motion in the simulation is in good agreement with by the UXRD measurement.

The fluence dependent study in Fig. 1 shows that for lower fluence the lattice stress starts about 130 fs later, yielding an estimate of the electron-phonon coupling time. Although the absolute determination of the time zero is only accurate within $\pm 100 \mathrm{fs}$, the relative phase delay of $130 \pm 50 \mathrm{fs}$ is determined with sufficient accuracy. The same clear trend is observed in the oscillation phase of the all-optical signal at $670 \mathrm{~nm}$ [Fig. 2(c)]. Below a fluence of about $20 \mathrm{~mJ} / \mathrm{cm}^{2}$ the electronic pressure is likely negligible compared to the lattice contribution to the expansion. Hence, below this fluence the phase is set by the electron-phonon coupling time in SRO. In the simplest models for metals, the electronic heat capacity rises linearly with the temperature and the lattice contribution 
saturates to the Doulong-Petit limit. However, calculations based on the electronic density of states (DOS) show that for most metals this is not true under strong nonequilibrium conditions. ${ }^{39}$ Since for the case of the bad metal SRO with strong electron correlations, simulations of the DOS strongly depend on the method used, ${ }^{40}$ and we do not attempt to predict the electronic stress contributions.

In the semiconducting material InSb a phase shift of the oscillations towards earlier times was observed for higher fluence. ${ }^{2}$ This was attributed to a decreasing lattice heating time for strong excitation, essentially because the reduced lifetime of LO phonons ${ }^{41}$ limits the carrier-lattice thermalization dynamics. ${ }^{42}$ Similar arguments could apply in the case of SRO. Specifically, the observation of an oscillation starting earlier for higher fluence is in contrast to the observation of electronic pressure in elementary metallic systems such as aluminum and gold. ${ }^{5,6}$ In these metals a larger electron-phonon coupling time leads to larger effect size in the opposite direction, namely because the electron-lattice heat transfer takes longer for high fluence. We cannot rule out that contributions from impulsive Raman scattering play a role in the phase shift; however, all processes in question-Raman, electronic pressure, lattice heat expansion-scale linearly with the pump fluence in the simplest models and would not explain a fluence dependence of the phase. Therefore we think that time- and temperaturedependent modification of the electron-phonon interaction must be responsible for the observed phase delay.

In conclusion, we have presented ultrafast x-ray diffraction (UXRD) and all-optical pump-probe experiments on the metal-dielectric superlattice STO/SRO. Only the combination of both methods allows one to experimentally deduce the absolute phase of the optically induced lattice motion. From fluence-dependent UXRD we extract a relative shift of the oscillation phase of the lattice which is readily ascribed to electronic pressure. The same fluence dependence of the phase is observed in all-optical experiments. However, the complex wavelength dependence of the broadband data highlight that the oscillation phase of all-optical experiments must be interpreted with care. Our x-ray calibrated simulations of the all-optical data validates the detailed interpretation.

\section{ACKNOWLEDGMENTS}

We thank R. van der Veen for her valuable experimental support at the MicroXAS-FEMTO beamline. We gratefully acknowledge the financial support by the BMBF via Grant No. 03WKP03A and the Deutsche Forschungsgemeinschaft (DFG) (Grant No. BA2281/3-1). *bargheer@uni-potsdam.de

${ }^{1}$ A. Morak, T. Kaempfer, I. Uschmann, A. Luebeke, E. Foerster, and

R. Sauerbrey, Phys. Status Solidi B 243, 2728 (2006).

${ }^{2}$ F. S. Krasniqi, S. L. Johnson, P. Beaud, M. Kaiser, D. Grolimund, and G. Ingold, Phys. Rev. B 78, 174302 (2008).

${ }^{3}$ P. Sondhauss, O. Synnergren, T. N. Hansen, S. E. Canton, H. Enquist, A. Srivastava, and J. Larsson, Phys. Rev. B 78, 115202 (2008).

${ }^{4}$ D. M. Fritz, D. A. Reis, B. Adams, R. A. Akre, J. Arthur, C. Blome, P. H. Bucksbaum, A. L. Cavalieri, S. Engemann, S. Fahy et al., Science 315, 633 (2007).

${ }^{5}$ J. Li, R. Clinite, X. Wang, and J. Cao, Phys. Rev. B 80, 014304 (2009).

${ }^{6}$ M. Nicoul, U. Shymanovich, A. Tarasevitch, D. von der Linde, and K. Sokolowski-Tinten, Appl. Phys. Lett. 98, 191902 (2011).

${ }^{7}$ A. Bartels, T. Dekorsy, H. Kurz, and K. Köhler, Phys. Rev. Lett. 82, 1044 (1999).

${ }^{8}$ H. J. Zeiger, J. Vidal, T. K. Cheng, E. P. Ippen, G. Dresselhaus, and M. S. Dresselhaus, Phys. Rev. B 45, 768 (1992).

${ }^{9}$ C.-K. Sun, J.-C. Liang, and X.-Y. Yu, Phys. Rev. Lett. 84, 179 (2000).

${ }^{10}$ T. E. Stevens, J. Kuhl, and R. Merlin, Phys. Rev. B 65, 144304 (2002).

${ }^{11}$ T. Dekorsy, G. Cho, and H. Kurz, in Light Scattering in Solids VIII, Vol. 76 of Topics in Applied Physics, edited by M. Cardona and G. Güntherodt (Springer, Berlin, 2000), pp. 169-209.

${ }^{12}$ V. V. Kruglyak, R. J. Hicken, G. P. Srivastava, M. Ali, B. J. Hickey, A. T. G. Pym, and B. K. Tanner, Phys. Rev. B 76, 012301 (2007).
${ }^{13}$ M. Hase, K. Ishioka, J. Demsar, K. Ushida, and M. Kitajima, Phys. Rev. B 71, 184301 (2005).

${ }^{14}$ S. L. Johnson, P. Beaud, C. J. Milne, F. S. Krasniqi, E. S. Zijlstra, M. E. Garcia, M. Kaiser, D. Grolimund, R. Abela, and G. Ingold, Phys. Rev. Lett. 100, 155501 (2008).

${ }^{15}$ M. Hase, M. Kitajima, A. Constantinescu, and H. Petek, Nature (London) 426, 51 (2003).

${ }^{16}$ A. Cavalleri, S. Wall, C. Simpson, E. Statz, D. W. Ward, K. A. Nelson, M. Rini, and R. W. Schoenlein, Nature (London) 442, 664 (2006).

${ }^{17}$ K. J. Yee, Y. S. Lim, T. Dekorsy, and D. S. Kim, Phys. Rev. Lett. 86, 1630 (2001).

${ }^{18}$ C. Colvard, T. A. Gant, M. V. Klein, R. Merlin, R. Fischer, H. Morkoc, and A. C. Gossard, Phys. Rev. B 31, 2080 (1985).

${ }^{19}$ M. Bargheer, N. Zhavoronkov, Y. Gritsai, J. C. Woo, D. S. Kim, M. Woerner, and T. Elsaesser, Science 306, 1771 (2004).

${ }^{20}$ M. Herzog, D. Schick, W. Leitenberger, R. Shayduk, R. M. van der Veen, C. J. Milne, S. L. Johnson, I. Vrejoiu, and M. Bargheer, New J. Phys. 14, 013004 (2012).

${ }^{21}$ C. von Korff Schmising, A. Harpoeth, N. Zhavoronkov, Z. Ansari, C. Aku-Leh, M. Woerner, T. Elsaesser, M. Bargheer, M. Schmidbauer, I. Vrejoiu et al., Phys. Rev. B 78, 060404 (2008).

${ }^{22}$ M. Herzog, W. Leitenberger, R. Shayduk, R. van der Veen, C. J. Milne, S. L. Johnson, I. Vrejoiu, M. Alexe, D. Hesse, and M. Bargheer, Appl. Phys. Lett. 96, 161906 (2010).

${ }^{23}$ P. Beaud, S. L. Johnson, A. Streun, R. Abela, D. Abramsohn, D. Grolimund, F. S. Krasniqi, T. Schmidt, V. Schlott, and G. Ingold, Phys. Rev. Lett. 99, 174801 (2007).

${ }^{24}$ The strain amplitude is derived by comparing the model and experimental data in the inset of Fig. 3(a) in Ref. 20. Since the agreement is nearly perfect, the mapping of fluence on strain yields 
the linear function presented in Fig. 1(b). We do not attempt to discuss the tiny deviations, as we focus here on the phase of the oscillations.

${ }^{25}$ I. Bozovic, M. Schneider, Y. Xu, R. Sobolewski, Y. H. Ren, G. Lüpke, J. Demsar, A. J. Taylor, and M. Onellion, Phys. Rev. B 69, 132503 (2004).

${ }^{26}$ S. Brivio, D. Polli, A. Crespi, R. Osellame, G. Cerullo, and R. Bertacco, Appl. Phys. Lett. 98, 211907 (2011).

${ }^{27}$ D. Schick, A. Bojahr, M. Herzog, C. von Korff Schmising, R. Shayduk, W. Leitenberger, P. Gaal, and M. Bargheer, Rev. Sci. Instrum. 83, 025104 (2012).

${ }^{28}$ F. Zamponi, Z. Ansari, C. von Korff Schmising, P. Rothhardt, N. Zhavoronkov, M. Woerner, T. Elsaesser, M. Bargheer, T. Trobitzsch-Ryll, and M. Haschke, Appl. Phys. A 96, 51 (2009).

${ }^{29}$ F. Zamponi, Z. Ansari, M. Woerner, and T. Elsaesser, Opt. Express 18, 947 (2010).

${ }^{30}$ C. Reich, P. Gibbon, I. Uschmann, and E. Förster, Phys. Rev. Lett. 84, 4846 (2000).

${ }^{31}$ C. von Korff Schmising, M. Bargheer, M. Kiel, N. Zhavoronkov, M. Woerner, T. Elsaesser, I. Vrejoiu, D. Hesse, and M. Alexe, Appl. Phys. B 88, 1 (2007).
${ }^{32}$ M. Woerner, C. von Korff Schmising, M. Bargheer, N. Zhavoronkov, I. Vrejoiu, D. Hesse, M. Alexe, and T. Elsaesser, Appl. Phys. A 96, 83 (2009).

${ }^{33}$ M. Herzog, D. Schick, P. Gaal, R. Shayduk, C. von Korff Schmising, and M. Bargheer, Appl. Phys. A 106, 489 (2012).

${ }^{34}$ R. Shayduk, H. A. Navirian, W. Leitenberger, J. Goldshteyn, I. Vrejoiu, M. Weinelt, P. Gaal, M. Herzog, C. von Korff Schmising, and M. Bargheer, New J. Phys. 13, 093032 (2011).

${ }^{35}$ M. Cardona, Phys. Rev. 140, A651 (1965).

${ }^{36}$ A. Giardini, J. Opt. Soc. Am. 47, 726 (1957).

${ }^{37}$ M. L. Hassel Ledbetter and S. Kim, Phase Transitions 23, 61 (1990).

${ }^{38}$ P. Kostic, Y. Okada, N. C. Collins, Z. Schlesinger, J. W. Reiner, L. Klein, A. Kapitulnik, T. H. Geballe, and M. R. Beasley, Phys. Rev. Lett. 81, 2498 (1998).

${ }^{39}$ Z. Lin, L. V. Zhigilei, and V. Celli, Phys. Rev. B 77, 075133 (2008).

${ }^{40}$ H.-T. Jeng, S.-H. Lin, and C.-S. Hsue, Phys. Rev. Lett. 97, 067002 (2006).

${ }^{41}$ K. T. Tsen, J. G. Kiang, D. K. Ferry, and H. Morkoc, Appl. Phys. Lett. 89 (2006).

${ }^{42}$ A. H. Chin, R. W. Schoenlein, T. E. Glover, P. Balling, W. P. Leemans, and C. V. Shank, Phys. Rev. Lett. 83, 336 (1999). 